\title{
Anti-Zeno effect in parametric down-conversion
}

\author{
A. Luis and L. L. Sánchez-Soto \\ Departamento de Óptica, Facultad de Ciencias Físicas, Universidad Complutense, 28040 Madrid, Spain
}

(Received 5 August 1997)

\begin{abstract}
Two examples are presented where the observation of the emission in parametric down-conversion leads to its enhancement instead of its inhibition. The improvement is analyzed in terms of the quantum features of the observation. [S1050-2947(98)04702-7]
\end{abstract}

PACS number(s): 03.65.Bz, 42.50.Dv

\section{INTRODUCTION}

The observation of a dynamical system can affect its evolution, especially in quantum theory. One of the consequences of this influence can be the inhibition of the isolated evolution of the system when its observation is attempted [1]. This is called the Zeno effect. The observation is usually described by repeated measurements performed in order to discover whether the initial state has changed or not [2]. In the limit of very frequent measurements it may happen that the system is locked in its initial state and the evolution, which was the aim of the observation, is in fact inhibited. This has been studied in a variety of processes such as atomic transitions [3], double-well potentials [4], and neutron spin dynamics [5].

In the first derivation of the Zeno effect, the statereduction postulate was used [1]. Since then, other purely dynamical approaches have been presented [4-6]. To observe the evolution of a system its coupling with a measuring apparatus is necessary. This usually implies its interaction with other degrees of freedom, although sometimes meaningful information can be obtained by some subtle modifications. In principle, this coupling disturbs the observed system. This dynamical stage of a measurement process appears to be enough to account for the Zeno effect. It occurs irrespective of whether the measurement is finally carried out or not: it is sufficient that it could be made.

In a purely dynamical explanation of the Zeno effect the inhibition of the original evolution is not a mandatory consequence. This opens the possibility of arranging the observation in order to enhance it, which may be called the antiZeno effect. This can be achieved by combining two opposite effects on the system. For instance, we can disturb previously the system so that the observation cancels the first impediment. The original dynamics would effectively occur at the same time it is observed, so the usual form of the Zeno effect would be avoided. Examples of evolution controlled by observation are known [7].

We might expect that if the observation is actually effective, the original evolution will not be recovered completely. Even in this case, it would be interesting to examine the limit of proximity to the original evolution and the way it depends on the quantum details of the observation.

Here we will examine these questions by using two examples of the Zeno effect in parametric down-conversion. The process under observation is the simultaneous emission of a pair of photons (twin photons) by spontaneous paramet- ric down-conversion in a nonlinear crystal. One of the emitted photons is evidence of the emission of the other. This can be used so as to infer when the emission of the other photon has taken place.

It has been shown that this leads to the inhibition of emission in modes satisfying conditions of perfect phase matching [8]. Here we will focus on those modes for which the emission is originally impeded by the phase mismatching. We will study whether the emission in these modes is improved by the modifications that make possible the observation. The degree of enhancement and the way it depends on the quantum character of the process will be examined as well.

In Sec. II we will briefly recall the isolated or unobserved parametric down-conversion together with a scheme to infer the moment of emission. This is the slicing of the crystal into a given number of pieces. In Sec. III we will consider a Kerr interaction coupling one of the down-converted modes with an auxiliary beam carrying the information concerning the moment of emission.

\section{OBSERVATION OF THE EMISSION BY DETECTION OF THE TWIN PHOTON}

First we will briefly recall the isolated or unobserved dynamics of the spontaneous parametric down-conversion (Fig. 1). A nonlinear crystal of length $L$ is pumped by a strong, classical, and coherent field to produce pairs of twin photons in signal, $a_{s}$, and idler, $a_{i}$, modes that are in vacuum before entering the crystal.

Since we are interested in field modes originally inhibited by the phase mismatch, we consider an appropriate mode selection performed by suitably placing filters and dia-

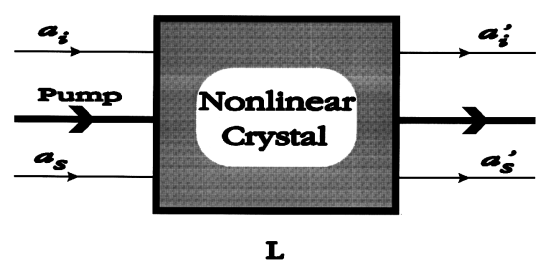

FIG. 1. Outline of a parametric down-conversion scheme with a crystal of length $L$, showing the input $a_{s}, a_{i}$ and output $a_{s}^{\prime}, a_{i}^{\prime}$ complex amplitude operators for the signal and idler fields. Beams have been represented parallel for simplicity. 
phragms. We assume that the modes selected satisfy the frequency-resonance condition $\omega_{p}=\omega_{s}+\omega_{i}$, where $\omega_{p}, \omega_{s}$, and $\omega_{i}$ are the frequencies of the pump, signal, and idler beams, respectively. We consider as well that the mode selection is so arranged that the dominant contribution is given by a pair of modes satisfying a perfect phase matching in the $X$ and $Y$ directions $\left(\Delta k_{x}=\Delta k_{y}=0\right)$ but having some mismatch in the $Z$ direction $\Delta k_{z}=k_{p, z}-k_{s, z}-k_{i, z}$ where $\Delta \mathbf{k}$ $=\mathbf{k}_{p}-\mathbf{k}_{s}-\mathbf{k}_{i}$ and $\mathbf{k}_{p}, \mathbf{k}_{s}$, and $\mathbf{k}_{i}$ are the corresponding wave vectors.

Assuming that we can neglect the pump depletion, the slowly varying complex amplitudes of the signal and idler beams satisfy the equations [9]

$$
\frac{d a_{s}}{d z}=-i \chi e^{-i \Delta k_{z} z} a_{i}^{\dagger}, \quad \frac{d a_{i}}{d z}=-i \chi e^{-i \Delta k_{z} z} a_{s}^{\dagger},
$$

where $\chi$ is a coupling parameter depending on the pumping field and the nonlinear characteristics of the medium. This parametric process is conveniently described by the effective interaction Hamiltonian [10]

$$
H=\hbar g\left(a_{s}^{\dagger} a_{i}^{\dagger} e^{-i \delta \Omega t}+a_{s} a_{i} e^{i \delta \Omega t}\right),
$$

where $g$, assumed to be real, is a constant proportional to $\chi$. The parameter $\delta \Omega$ and the effective interaction time in the crystal $\tau$ are given by

$$
\delta \Omega \tau=\Delta k_{z} L
$$

We will consider also the short-time regime $g \tau \ll 1$.

To first order in $g \tau$, the output complex amplitude of the signal mode $a_{s}^{\prime}$ is related to the input ones by

$$
a_{s}^{\prime} \simeq a_{s}-i g \tau \frac{\sin (\delta \Omega \tau / 2)}{\delta \Omega \tau / 2} e^{-i \delta \Omega \tau / 2} a_{i}^{\dagger} .
$$

The initial field state will be always the vacuum in both modes. The first-order approximation in $g \tau$ means that the probability of emission of more than one pair of twin photons is negligible. The probability of finding one output signal photon is

$$
P \simeq(g \tau)^{2}\left[\frac{\sin (\delta \Omega \tau / 2)}{\delta \Omega \tau / 2}\right]^{2}
$$

This expression reflects the impediment of down-conversion by phase mismatch. Maximum emission $P_{\max } \simeq(g \tau)^{2}$ occurs provided $\delta \Omega=0$.

The emission of the signal photon is always accompanied by the emission of a twin photon in the idler mode. The entangled nature of this photon pair has been utilized hitherto in a number of fundamental experiments in quantum optics [11]. In our context, it serves to detect the emission of the signal photon without apparently disturbing or interrupting the signal path within the crystal.

In this section we study a first and simple implementation of this possibility, which is schematized in Fig. 2. The original crystal is divided into $N$ identical pieces of length $\delta L$ $=L / N$ (the associated interaction time in each piece being $\delta \tau=\tau / N)$. To simplify the analysis as far as possible, we can assume that the signal beams of consecutive slices are per-

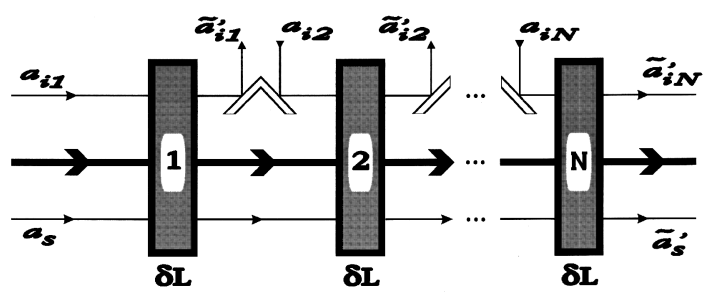

FIG. 2. Modified scheme to infer the moment of emission and consisting of $N$ crystals of length $\delta L=L / N$. After each piece the output idler beams $\tilde{a}_{i, k}^{\prime}$ are removed by mirrors inserted in the idler path and replaced by different input idler fields $a_{i, k}$ in vacuum. Beams have been represented parallel for simplicity.

fectly superimposed and aligned. Assuming that reflections or any other disturbance of the signal beam are negligible, the signal path after the slicing is indistinguishable from the original one in the full crystal of Fig. 1. On the other hand, the idler beams after each slab are removed by mirrors and replaced by different input fields in vacuum. Detectors could be placed at the output idler paths to detect the emission. If the idler photon is detected after one of the pieces we can infer that the signal photon has been emitted somewhere within the same piece. In this way, the moment of emission of the signal photon can be inferred with an accuracy of the order of $R=\tau / \delta \tau=N$.

If photons were emitted according to classical probabilities, this detection should not affect the emission itself, because the signal photon has already been generated. However, in a quantum process probability amplitudes are more relevant than probabilities. Next we examine the way this arrangement modifies the emission. Since a different vacuum mode is at the idler input of each piece, the total input-output relation results from the consecutive application of $N$ transformations of the form (2.4) with $\delta \tau$ instead of $\tau$ and a new input idler mode $a_{i, m}$ each time. The output signal complex amplitude $\widetilde{a}_{s}^{\prime}$ is

$$
\widetilde{a}_{s}^{\prime} \simeq a_{s}-i g \delta \tau \frac{\sin (\delta \Omega \delta \tau / 2)}{\delta \Omega \delta \tau / 2} e^{-i \delta \Omega \delta \tau / 2} \sum_{m=1}^{N} a_{i, m}^{\dagger}
$$

where, for simplicity, we have dismissed the free propagation between pieces. The probability of emission of the signal photon becomes now

$$
\widetilde{P} \simeq(g \tau)^{2} \frac{1}{N}\left[\frac{\sin (\delta \Omega \tau / 2 N)}{\delta \Omega \tau / 2 N}\right]^{2}
$$

which can be compared with the probability of the isolated or unobserved situation in Eq. (2.5). In Fig. 3(a) we have represented $\widetilde{P} / P_{\max }$ as a function of $\delta \Omega \tau$ and $N$.

In Eq. (2.7) we can notice the competition of two different effects. In the first place, there is a term $1 / N$ that tends to decrease the emission irrespective of the phase mismatch. This inhibition is stronger as $N$ increases, i.e., when increasing the accuracy of the observation. This is the only contribution when $\delta \Omega=0$, and is the form of the Zeno effect, which results from the first-order treatment in $g \tau$ performed here. 

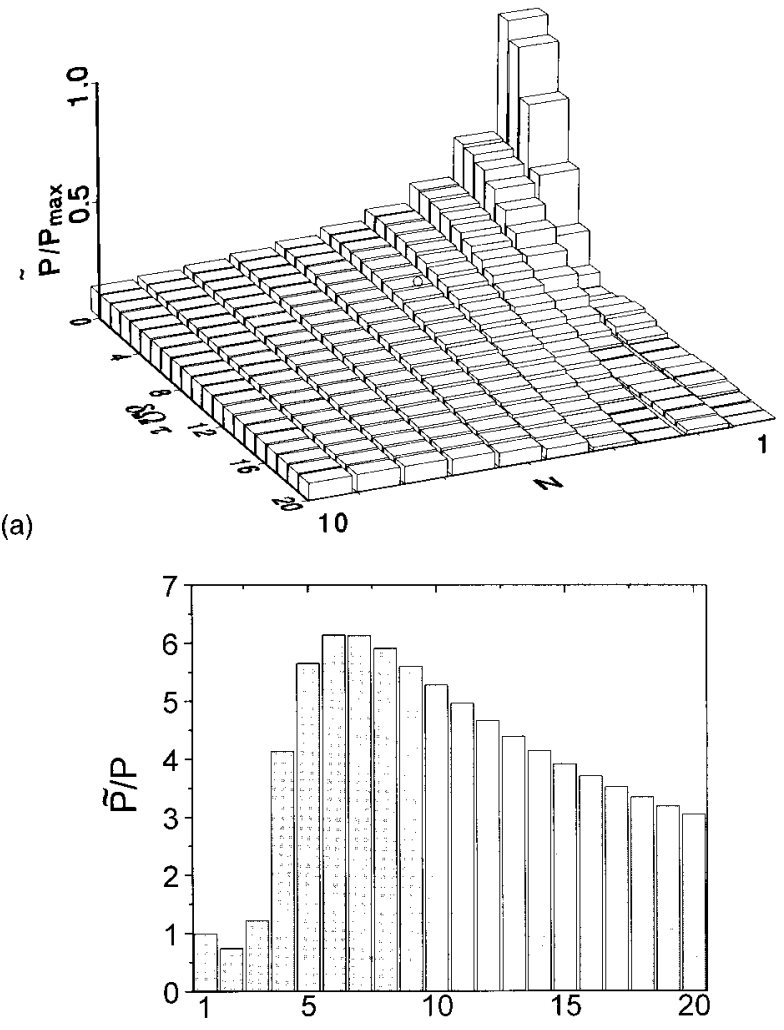

(b)

N

$$
\widetilde{P}_{\max } \simeq(g \tau)^{2} \frac{1.449}{\delta \Omega \tau}=(g \tau)^{2} \frac{0.622}{N_{\max }}=(g \tau)^{2} \frac{0.622}{R}
$$

We can observe that this value is greater than $P$. This is because $P$ is proportional to $1 /(\delta \Omega \tau)^{2}$ while $\widetilde{P}_{\max }$ is proportional to $1 /(\delta \Omega \tau)$. Even in the case when $\sin (\delta \Omega \tau / 2) \simeq 1$ we have

$$
\frac{\widetilde{P}_{\max }}{P} \simeq \delta \Omega \tau \simeq N_{\max }=R .
$$

The improvement is as large as the phase mismatch. In Fig. 3(b) we have represented $\widetilde{P} / P$ as a function of $N$ for $\delta \Omega \tau$ 15. Maximum occurs for $N_{\max }=6$, giving $\widetilde{P}_{\max }$ $\simeq 0.096(g \tau)^{2}$ while $P \simeq 0.016(g \tau)^{2}$, so $\widetilde{P}_{\max } / P \simeq 6$. For $N$ $>N_{\max }$ it can be appreciated that $\widetilde{P}$ scales as $1 / N$.

The disturbance introduced in order to make possible the observation can effectively remove the mismatch, with the corresponding enhancement of the emission. This can be seen by comparing $\widetilde{P}_{\max }$ with the probability of emission in modes with perfect phase matching. If $\delta \Omega=0$ in Eq. (2.7), we have $\widetilde{P} \simeq(g \tau)^{2} / R$, which, except for the numerical factor, coincides with $\widetilde{P}_{\max }$ in Eq. (2.10).

This behavior can be understood in quantum terms if we regard the probability of emission as the result of the interference of probability amplitudes. The emission in the unobserved case results from the coherent superposition of probability amplitudes originated in each part of the crystal. They are coherent as long as they can be regarded as being stimulated by the same input vacuum that imparts phase correlations between them $[8,12]$. Due to the mismatch, this interference is partially or completely destructive depending on $\delta \Omega \tau$. After interrupting $N$ times the idler mode, the probability amplitudes from each piece are mutually incoherent since they are stimulated by different vacuum modes. When $N<N_{\text {max }}$, this loss of coherence prevents destructive interference and the probability of emission increases. When $N$ is close to $N_{\max }$ the slicing of the crystal has almost completely removed the mismatch. But the mutual incoherence of the $N$ emitters prevents any kind of constructive interference and the maximum emission $\widetilde{P}_{\max }$ is always less than $P_{\max }$. When $N>N_{\max }$ the induced incoherence continues and the probability of emission decreases as $1 / N$.

A classical analog of this quantum interference can be found in Fraunhofer diffraction, provided that probability amplitudes are translated into field amplitudes and probability of emission into intensity of the diffraction pattern. Equation (2.5) describes the diffraction by a slit of width $L$ illuminated by a plane wave, where $\Delta k_{z}$ represents the position in the pattern relative to its maximum at $\Delta k_{z}=0$. Equation (2.7) is the diffraction pattern of $N$ slits of width $\delta L=L / N$ illuminated by mutually incoherent plane waves of the same amplitude. In this analogy we have the same competition between destructive interference and incoherence. which, more properly, should be understood as the nearest integer to this quantity. The maximum emission is 


\section{OBSERVATION OF THE EMISSION BY KERR INTERACTION}

Although the previous slicing of the crystal provides a clear illustration of the phenomenon, it might be regarded as a crude realization of the observation that demands severe assumptions. In this section we consider another scheme where the observation is controlled by an auxiliary field. This will illustrate the relevant features of the phenomenon without further simplifying assumptions.

We shall assume that within the crystal there is a Kerr interaction between the idler mode and an auxiliary field mode $b$. This coupling can be conveniently described by the effective interaction Hamiltonian

$$
\widetilde{H}=\hbar g\left(a_{s}^{\dagger} a_{i}^{\dagger} e^{-i \delta \Omega t}+a_{s} a_{i} e^{i \delta \Omega t}\right)+\hbar \kappa a_{i}^{\dagger} a_{i} b^{\dagger} b .
$$

Due to the Kerr interaction, the field $b$ will experience a phase change proportional to the length covered by the idler photon from it has been emitted until it leaves the crystal. Equivalently, the phase change is proportional to the time spent by the idler photon in the crystal. The moment of emission, or the region of the crystal where the emission has taken place, can be inferred by a phase-dependent measurement, like homodyne detection for example, of the output field in mode $b$.

Although the signal mode does not interact directly with the mode $b$, this arrangement also modifies the emission. In the short-time regime $g \tau \ll 1$, the input-output relation is

$$
\tilde{a}_{s}^{\prime} \simeq a_{s}-i g \tau \frac{\sin \left[\left(\kappa b^{\dagger} b-\delta \Omega\right) \tau / 2\right]}{\left(\kappa b^{\dagger} b-\delta \Omega\right) \tau / 2} e^{i\left(\kappa b^{\dagger} b-\delta \Omega\right) \tau / 2} a_{i}^{\dagger} .
$$

When the input idler and signal modes are in vacuum, the probability of emission of the signal photon is

$$
\begin{aligned}
\widetilde{P} & \simeq(g \tau)^{2}\left\langle\psi\left|\left\{\frac{\sin \left[\left(\kappa b^{\dagger} b-\delta \Omega\right) \tau / 2\right]}{\left(\kappa b^{\dagger} b-\delta \Omega\right) \tau / 2}\right\}^{2}\right| \psi\right\rangle \\
& =(g \tau)^{2} \sum_{n=0}^{\infty}\left\{\frac{\sin [(\kappa n-\delta \Omega) \tau / 2]}{(\kappa n-\delta \Omega) \tau / 2}\right\}^{2} P_{\psi}(n),
\end{aligned}
$$

where $|\psi\rangle$ is the input field state in mode $b$ and $P_{\psi}(n)$ its photon-number distribution.

We can observe in Eq. (3.3) that the coupling of the observation arrangement with the observed system has the form of a mismatch that depends on the photon number $b^{\dagger} b$. This will modify the original distribution of the emission between modes established by the original mismatch $\Delta k_{z}$. This new balance will increase the emission in modes previously impeded. As in the previous section, we will focus on modes inhibited by the original phase mismatch, which can be selected by properly arranging filters and pinholes.

The probability of emission and the performance of the observation strongly depend on the field state $|\psi\rangle$. To understand how emission and observation are controlled by $|\psi\rangle$, let us assume momentarily that the field mode $b$ could be described classically with nonfluctuating intensity and phase. From Eq. (3.3), we can see that a suitable choice of the intensity would cancel completely the effect of the mismatch, achieving the maximum emission $P_{\max }$ allowed by the original nonlinear interaction. On the other hand, if there were no phase fluctuations, the moment of emission would be inferred with arbitrary accuracy.

In the quantum case, phase and number fluctuations prevent reaching these two goals simultaneously. A measure of the uncertainty in the phase-change estimation after a single observation can be given by the phase dispersion $\Delta \phi$ in the state $|\psi\rangle$ [13]. Under appropriate conditions for a relevant phase estimation, $\Delta \phi$ and the photon-number uncertainty $\Delta n$ satisfy the relation $\Delta \phi \Delta n \geqslant 1 / 2$ [14]. As in Sec. II, the probability of emission results from the competition of two opposite effects: precise cancellation of the mismatch and resolution of the observation, although the underlying quantum mechanism is slightly different.

Precise cancellation of the mismatch requires small $\Delta n$ around an optimum value of the mean photon number, but in such a case the measurement will provide no information because of the large phase uncertainty. For example, this occurs when the input state in mode $b$ is a number state $|n\rangle$. If $\kappa \tau n \simeq \delta \Omega \tau$ then $\widetilde{P} \simeq(g \tau)^{2}$, and the emission takes its maximum value. However, as a matter of fact, there is no observation at all because number states have completely random phase, and so no phase change can be detected. Vice versa, a relevant inference of the moment of emission requires large photon-number fluctuations but, as can be seen in Eq. (3.3), large $\Delta n$ will prevent a precise cancellation of the mismatch.

We can see that the phase-number uncertainty relation is translated into a complementarity between the probability of emission and the capability of determining when emission occurs. The initial state of the field $|\psi\rangle$ establishes a particular balance between these two complementary quantities.

In what follows we look for a quantitative formulation of these points. We assume that $|\psi\rangle$ is appropriate for inferring the moment of emission. The phase change $\phi$ that would experience the state $|\psi\rangle$ is of the order of $\kappa \tau$, which can be considered as a small number. The resolution in the phase change is $R=\phi / \Delta \phi=\tau / \delta \tau \leqslant 2 \kappa \tau \Delta n$. A meaningful observation requires large $\Delta n$ and, accordingly, the mean photon number $\bar{n}$ has to be large enough. Under these conditions we will examine whether the emission improves.

To this end, we have to evaluate Eq. (3.3), looking for its maximum under the previous conditions. First, we transform Eq. (3.3) into another expression more suited for calculation. It can be checked that

$$
\begin{aligned}
\widetilde{P} & \simeq(g \tau)^{2} \sum_{n=0}^{\infty}\left\{\frac{\sin [(\kappa n-\delta \Omega) \tau / 2]}{(\kappa n-\delta \Omega) \tau / 2}\right\}^{2} P_{\psi}(n) \\
& =(g \tau)^{2} \int_{-1}^{1} d x(1-|x|) e^{-i \delta \Omega \tau x} C(\kappa \tau x),
\end{aligned}
$$

where $C(\kappa \tau x)$ is the photon-number characteristic function [10]

$$
C(\kappa \tau x)=\left\langle\psi\left|e^{i \kappa \tau x b^{\dagger} b}\right| \psi\right\rangle
$$


If an accurate determination of the phase shift is desired, then $C(\kappa \tau x)$ should be peaked around $x=0$. This is because the states $e^{i \kappa \tau x b^{\dagger} b}|\psi\rangle$ are different enough for different $x$ and will be easily distinguished by measurement. The quantity $|C(\phi)|$ has been used to determine how accurately a phase change in the state $|\psi\rangle$ can be measured [15].

To evaluate Eq. (3.4) we will assume a Gaussian for $C(\kappa \tau x)$. Although this is a particular case, we think it is representative enough, since it provides a useful approximation for coherent as well as squeezed states of high intensity and moderate squeezing. According to the general relation between photon-number characteristic function and cumulants [10], the form of $C(\kappa \tau x)$ must be

$$
C(\kappa \tau x) \simeq e^{i \kappa \tau \bar{n} x} e^{-(\kappa \tau \Delta n)^{2} x^{2} / 2}
$$

We have to compute

$$
\widetilde{P} \simeq(g \tau)^{2} \int_{-1}^{1} d x(1-|x|) e^{i(\kappa \tau \bar{n}-\delta \Omega \tau) x} e^{-(\kappa \tau \Delta n)^{2} x^{2} / 2} .
$$

For fixed $\Delta n$, maximum $\widetilde{P}$ requires the equality $\kappa \tau \bar{n}$ $=\delta \Omega \tau$. This is the optimum value for the mean photon number that completely cancels the mismatch. In such a case, the probability of emission depends only on the photon-number fluctuations

$$
\widetilde{P}_{\max } \simeq(g \tau)^{2} \int_{-1}^{1} d x(1-|x|) e^{-(\kappa \tau \Delta n)^{2} x^{2} / 2},
$$

giving

$$
\begin{aligned}
\widetilde{P}_{\max } \simeq & (g \tau)^{2}\left\{\frac{\sqrt{2 \pi}}{\kappa \tau \Delta n} \operatorname{erf}(\kappa \tau \Delta n / \sqrt{2})\right. \\
& \left.+\frac{2}{(\kappa \tau \Delta n)^{2}}\left[e^{-(\kappa \tau \Delta n)^{2} / 2}-1\right]\right\},
\end{aligned}
$$

where erf is the error function.

Meaningful resolution requires large $\kappa \tau \Delta n$, so $\widetilde{P}_{\max }$ can be properly replaced by

$$
\widetilde{P}_{\max } \simeq(g \tau)^{2} \frac{\sqrt{2 \pi}}{\kappa \tau \Delta n} .
$$

This is the maximum probability attainable under these conditions. We can assume that the input state is optimized in the sense that $\Delta \phi \Delta n \simeq 1 / 2$. This means that for given $\Delta \phi$, it has minimum photon-number fluctuations allowed by the uncertainty relation and, therefore, it provides maximum emission for a given resolution. The equality in the uncertainty relation gives $R \simeq 2 \kappa \tau \Delta n$ and then $\widetilde{P}_{\max }$ can be written as

$$
\widetilde{P}_{\max } \simeq(g \tau)^{2} 2 \frac{\sqrt{2 \pi}}{R} .
$$

This means an effective improvement of emission. Even when in Eq. (2.5) $\sin (\delta \Omega \tau / 2) \sim 1$, we have

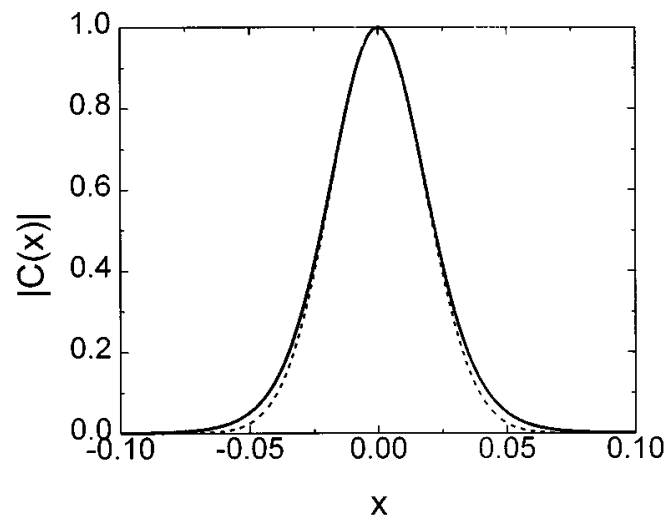

FIG. 4. Modulus of the photon-number characteristic function for a squeezed state with $\bar{n}=150, r=1.5$, and $\delta=\pi / 2$ (solid line) together with its Gaussian approximation (dashed line).

$$
\frac{\widetilde{P}_{\max }}{P} \simeq \delta \Omega \tau \frac{\bar{n}}{\Delta n} \simeq\left(\frac{\bar{n}}{\Delta n}\right)^{2} R,
$$

where the relations $\kappa \tau \bar{n}=\delta \Omega \tau$ and $R \simeq 2 \delta \Omega \tau \Delta n / \bar{n}$ have been used. The emission with observation is larger than without observation by a factor at least of the order of $R$.

Also in this scheme the enhancement of emission cannot be arbitrary and is again limited by the resolution achieved, as shown in Eq. (3.11).

These equations can serve to express quantitatively our previous discussion concerning complementarity between probability of emission and resolution. The upper bound (3.11) means the following inequality:

$$
R \widetilde{P} \leqslant 2 \sqrt{2 \pi} P_{\max }
$$

The equality is reached when $\kappa \tau \bar{n}=\delta \Omega \tau$, which is the translation to these quantities of the phase-number uncertainty relation, since $R \propto 1 / \Delta \phi$ and $\widetilde{P} \propto 1 / \Delta n$.

These points may be illustrated by an example. We consider modes with a phase mismatch $\delta \Omega \tau=15$ and $\kappa \tau=0.1$. The condition $\kappa \tau \bar{n}=\delta \Omega \tau$ leads to $\bar{n}=150$ as the mean photon number providing maximum emission. Since $\Delta n$ has to be as large as possible, we consider as input state in mode $b$ the squeezed state

$$
|\psi\rangle=e^{\alpha b^{\dagger}-\alpha^{*} b} e^{\xi^{*} b^{2}-\xi b^{\dagger 2}}|0\rangle,
$$

where $|0\rangle$ is the vacuum. This state has

$$
\bar{n}=|\alpha|^{2}+\sinh ^{2} r
$$

$$
(\Delta n)^{2}=|\alpha|^{2}\left(e^{-2 r} \cos ^{2} \delta+e^{2 r} \sin ^{2} \delta\right)+2 \sinh ^{2} r \cosh ^{2} r,
$$

where $r=2|\xi|$ and $\delta=\arg (\alpha)-\arg (\xi) / 2$. For example, when $\bar{n}=150, r=1.5$, and $\delta=\pi / 2$, we have $\Delta n=54.5, \kappa \tau \Delta n$ $=5.45$, and $R=11$. The number-phase uncertainty product is $\Delta \phi \Delta n=0.55$, which is very close to the minimum $1 / 2$. In Fig. 4 we have represented the modulus of the photonnumber characteristic function and its Gaussian approximation for this example, showing that they are very similar. The approximation (3.9) gives $\widetilde{P}_{\max } \simeq 0.3925(g \tau)^{2}$. 


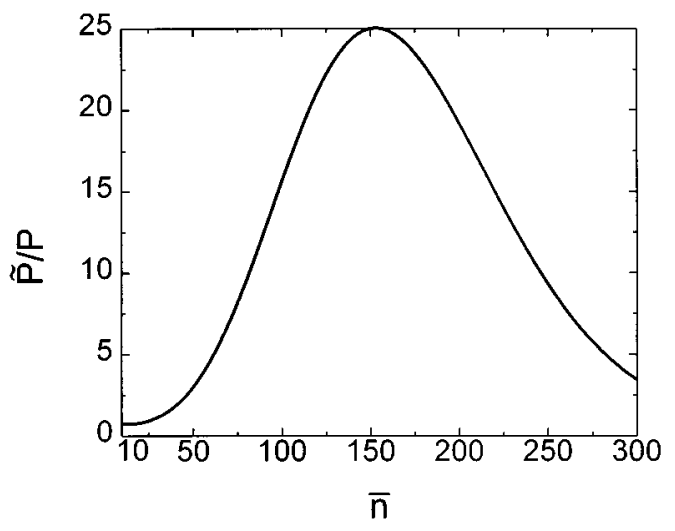

FIG. 5. Quotient between the probability of emission $\widetilde{P}$ in the Kerr arrangement and the probability $P$ of the unobserved parametric down-conversion as a function of the mean photon number $\bar{n}$ of an initial squeezed state with $r=1.5, \delta \Omega \tau=15$, and $\kappa \tau=0.1$.

In Fig. 5 we have plotted $\widetilde{P} / P$ using a numerical calculation of Eq. (3.3) as a function of the mean photon number of a squeezed state with $r=1.5$ and $\delta=\pi / 2$. This figure has a maximum for $\bar{n} \simeq 153$, the maximum emission being $0.3917(g \tau)^{2}$, that is 25 times larger than the probability of emission without observation. These values are very close to those obtained by means of the approximations developed here.

Finally, it is interesting to point out that the resolution achievable when maximum emission occurs is limited by the phase mismatch to $R=2 \delta \Omega \tau \Delta n / \bar{n}$. This is because the quantity $\Delta n / \bar{n}$ cannot be much larger than 1 , since the photon number is bounded from below and then $\Delta n$ and $\bar{n}$ are not independent. If we would replace $b^{\dagger} b$ by another field variable not bounded from below, like field quadratures, then $\Delta n$ and $\bar{n}$ would be replaced by truly independent variables. In such a case $R$ would not be bounded by the phase mismatch.

\section{CONCLUSIONS}

The dynamics of an observation arrangement leads to the possibility of controlling the evolution of the observed sys- tem. We have shown that there are situations where the observation of emission in parametric down-conversion leads to its enhancement instead of its inhibition. In our examples, the emission is initially impeded by the phase mismatch. One of the effects of the observation is the removal of this impediment with the corresponding enhancement. But this improvement has to compete with other disturbances of quantum origin. Under appropriate conditions this competition leads to an upper limit.

Two schemes of observation have been analyzed. They lead to similar results, although the underlying quantum mechanisms are different. In the first example, the emission is finally limited by the incoherent superposition of probability amplitudes. In the second example, the explanation can be found in the phase-number uncertainty relation.

Nevertheless, it is possible to account for both examples simultaneously by means of a single argument, which explains why the same result is obtained in very different arrangements. In the quantum domain, interference is a manifestation of the intrinsic indistinguishability between different paths for the process to occur. Whenever paths become distinguishable, the interference is destroyed, leading to the superposition of probabilities instead of probability amplitudes. If the original interference is destructive, as was the case here, the final probability can be increased. But this has a limit, since it is not possible to convert destructive into constructive interference by these means.

It should be noticed that the points discussed throughout this work occur irrespective of whether the detection of the idler photon in Sec. II and the phase-dependent measurement in Sec. III are actually carried out or not. The modification of the system, which is responsible for its different dynamics, is previous to the final realization of the measurement, so the system has already changed irrespective of whether detectors are placed or not at the corresponding output beam.

\section{ACKNOWLEDGMENT}

We wish to thank Professor G. García-Alcaine for a careful reading of the manuscript.
[1] B. Misra and E. C. G. Sudarshan, J. Math. Phys. 18, 756 (1977).

[2] A. Peres, Quantum Theory: Concepts and Methods (Kluwer Academic, Dordrecht, 1993).

[3] R. J. Cook, Phys. Scr. T21, 49 (1988); W. M. Itano, D. J. Heinzen, J. J. Bollinger, and D. Wineland, Phys. Rev. A 41, 2295 (1990).

[4] T. P. Altenmüller and A. Schenzle, Phys. Rev. A 49, 2016 (1994).

[5] H. Nakazato, M. Namiki, S. Pascazio, and H. Rauch, Phys. Lett. A 199, 27 (1995); A. Venugopalan and R. Ghosh, ibid. 204, 11 (1995).

[6] S. Pascazio and M. Namiki, Phys. Rev. A 50, 4582 (1994).
[7] Y. Aharonov and M. Vardi, Phys. Rev. D 21, 2235 (1980); A. Peres and A. Ron, Phys. Rev. A 42, 5720 (1990); T. F. Jordan, E. C. G. Sudarshan, and P. Valanju, ibid. 44, 3340 (1991): T. P. Altenmüller and A. Schenzle, ibid. 48, 70 (1993).

[8] A. Luis and J. Peřina, Phys. Rev. Lett. 76, 4340 (1996).

[9] A. Yariv, Quantum Electronics (John Wiley, New York, 1975).

[10] J. Perina, Quantum Statisics of Linear and Nonlinear Optical Phenomena, 2nd ed. (Kluwer Academic, Dordrecht, 1991).

[11] J. Peřina, Z. Hradil, and B. Jurčo, Quantum Optics and Fundamentals of Physics (Kluwer Academic, Dordrecht, 1994).

[12] M. O. Scully and U. W. Rathe, Opt. Commun. 110, 373 (1994). 
[13] A. Bandilla, H. Paul, and H. H. Ritze, Quantum Opt. 3, 267 (1991); Z. Hradil, ibid. 4, 93 (1992): J. M. Lévy-Leblond, Ann. Phys. (N.Y.) 101, 319 (1976); T. Opatrný, J. Phys. A 27, 7201 (1994); A. Lukš and V. Peřinová, Czech. J. Phys. 41, 1205 (1991).
[14] A. Lukš and V. Peřinová, Phys. Rev. A 45, 6710 (1992): Phys. Scr. T48, 94 (1993).

[15] M. Hillery, M. Freyberger, and W. Schleich, Phys. Rev. A 51, 1792 (1995). 